\title{
Effect of Drosophila suzukii on blueberry VOCs: chemical cues for a pupal parasitoid, Trichopria anastrephae
}

Gerardo de la Vega ( $\sim$ delavega.gerardo@gmail.com )

IFAB (INTA-CONICET) https://orcid.org/0000-0003-3095-0077

\section{Federico Triñanes}

Universidad de la Republica Facultad de Quimica Laboratorio de Ecologia Quimica

Andres González

Universidad de la Republica Facultad de Quimica Laboratorio de Ecologia Quimica

\section{Research Article}

Keywords: spotted-wing drosophila, local parasitoids, VOCs blends, biocontrol agents

Posted Date: March 12th, 2021

DOI: https://doi.org/10.21203/rs.3.rs-320923/v1

License: (c) (i) This work is licensed under a Creative Commons Attribution 4.0 International License.

Read Full License

Version of Record: A version of this preprint was published at Journal of Chemical Ecology on July 17th, 2021. See the published version at https://doi.org/10.1007/s10886-021-01294-7. 


\section{Abstract}

Biocontrol agents such as parasitic wasps use long-range volatiles and host-associated cues from lower trophic levels to find their hosts. Although, this chemical landscape may be altered by the invasion of exotic insect species. The spotted-wing drosophila (SWD), Drosophila suzukii (Diptera: Drosophilidae), is a highly polyphagous fruit pest native to eastern Asia and recently arrived in South America. The aim of our study was to characterize the effect of SWD attack on the volatile organic compounds (VOCs) of blueberries, a common host fruit, and to correlate these odor changes with the olfactory-mediated behavioral response of resident populations of Trichopria anastrephae parasitoids. Using fruit VOC chemical characterization followed by multivariate analyses of the odor blends of SWD-attacked blueberries, we showed that the development of SWD immature stages inside the fruit generates a different odor profile than control fruits (physically damaged and free of damage). These differences can be explained by the diversity, frequency and amounts of fruit VOCs. The behavioral response of female parasitic wasps showed that $T$. anastrephae has an innate attraction to volatile cues from infested host fruits, which may lead to successful location of their insect host. Since resident parasitoids are able locate this novel potential host, biological control programs using local populations may be plausible as a SWD control strategy.

\section{Introduction}

Global trade network is one of the main causes of pest species range expansion. The constant flow of agricultural products among countries facilitate species to reach novel environments (Anderson et al. 2004, Lantschner et al. 2019), threatening not only the production of goods but also the local biodiversity (Harvey and Fortuna 2012). When exotic insect species arrive in local natural communities, the native food webs are most certainly altered, as is the entire ecosystem by cascading chemical, physiological and ecological changes across trophic levels (Chabaane et al. 2015). The presence or absence of antagonists (i.e. predators or parasitoids) is a key commponent in the success or failure of non-native species in a novel range, involving both top-down and bottom-up biotic influences among multiple trophic levels (Schultz et al. 2019). In this context, understanding how non-native pest species establish and interact with the native community is critical to forecast their success and to devise pest management strategies.

Chemical cues and signals are essential mediators in the ecological interactions of insects. Trophic interactions within native communities often rely on these cues, and are therefore susceptible to chemical landscape alterations that may result from the invasion of exotic insect species (Chabaane et al. 2015, Rombaut et al. 2017, Mair and Ruther 2019). A common third trophic level involved in plant defense are parasitic wasps -hymenopteran insects which immature life stages occur in or on other arthropods, mostly other insects- (Schultz et al. 2019, Cusumano et al. 2020). To find adequate habitats for potential mates or hosts, parasitic wasps use long-range volatiles and host-associated cues from lower trophic 
levels (Mumm and Hilker 2005, Desurmont et al. 2020). While searching for hosts, parasitic wasps need to integrate specific chemical cues with context background odors, which may be classified as irrelevant odors (no role in foraging behavior), masking odors (interfere and neutralize an attractive odor source), and enhancing odors (increase the attractiveness of an odor source) (Schröder and Hilker 2008). In this sense, mixtures of host-associated volatiles and background odors may be important mediators in tritrophic interactions involved in parasitoid host location (Schröder and Hilker 2008, Desurmont et al. 2020). In agricultural systems, these synergistic effects may be necessary to enhance the behavioral response of parasitic wasps to efficiently find their hosts (Liu et al. 2019).

Even though invasion ecology is a growing field, seldom studies focus on the effect of new exotic insects on multitrophic interactions in native communities (Chabaane et al. 2015). Our study system involves three trophic levels, a native population of the parasitoid wasp Trichopria anastrephae (Hymenoptera: Diapriidae), a potential new insect host that has recently arrived in South America, Drosophila suzukii (Matsumura) (Diptera: Drosophilidae), and one of its soft-skin fruit hosts, Vaccinium corymbosum (blueberries). Drosophila suzukii, known as the spotted wing drosophila (SWD), is a highly invasive insect native to eastern Asia (Walsh et al. 2011). In the last decade it became a risk for soft-skin fruits affecting a variety of cherry and berry crops (i.e. strawberries, blueberries) (Bolda et al. 2010; Walsh et al. 2011). SWD invasions were initially detected in Europe and North America in 2008 (Cini et al. 2012) and between 2012 and 2015 arrived in South America spreading from Brazil to the Patagonia region in southern Argentina (de la Vega and Corley 2019, de la Vega et al. 2020). The fly presents an important difference with most Drosophila species, which oviposit in decaying or overripe fruit; SWD females have a serrated ovipositor to pierce fruit skin, allowing them to lay eggs inside undamaged fresh and ripening fruit (Atallah et al. 2014). Before D. suzukii invaded agroecosystems, wounds on fruits such as grapes were due to climatic factors (i.e. hail, heat shock, heavy rain) or physical damages by birds or wasps (Rombaut et al. 2017). Consequently, the attack of SWD facilitates $D$. melanogasterinfestation. As a consequence, rather than competing with closely related species, the invader makes available otherwise non-accessible resources, hence opening a new ecological niche for native fructivorous insects (Rombaut et al. 2017).

Skin-soft fruits such as blueberries change their profile of volatile organic compounds (VOCs) as they ripen or senesce (Farneti et al. 2017). Therefore, fruit VOCs may potentially be used by D. melanogaster and $D$. suzukii to find different fruit stages (Keesay et al. 2015, Karageorgi et al. 2017). A host preference shift from rotten to fresh fruit has been proposed for SWD (Keesay et al. 2015), an ecological shift that may have had an impact on higher trophic levels as well. In this scenario, understanding multitrophic chemical ecology aspects related to the invasion of $D$. suzukii to new environments represents an opportunity to understand its effect on established populations of natural enemies. In turn, this understanding may result in improvements for integrated pest management programs. 
There is growing interest in the development of environmentally friendly pest management methods to reduce the application of harmful pesticides (Kruitwagen et al. 2018). Thus, at least four pupal parasitic wasps had been tested as biological control agents of SWD, Pachycrepoideus vindemiae (Rondani Hymenoptera: Pteromalidae); Spalangia erythromera (Förster - Hymenoptera: Pteromalidae); Trichopria drosophilae (Perkins - Hymenoptera: Diapriidae) and Trichopria anastrephae (Lima - Hymenoptera: Diapriidae). These were all able to parasitize D. suzukii under laboratory conditions (Ibouh et al. 2019, Vieira et al. 2019). While potential biological control agents may be identified in SWD's native range (Lee et al. 2019), complex international regulations and biodiversity risks associated with the introduction of exotic natural enemies underline the need for improving the efficacy of resident species of natural enemies (Kruitwagen et al. 2018). In this sense, the presence of $T$. anastrephae populations in Latin America has been reported since 2001 (summarized in Vieira 2019), although biological control studies against $D$. suzukii are still incipient in the region (i.e. Wollmann et al. 2016, Vieira et al. 2019), with no studies focusing on the chemical ecology of these interactions. In this particular system, understanding if parasitoids have innate ability to find and exploit SWD-infested fruit by using volatile cues bears potential implications for the biological control of this fruit pest. More specifically, if the parasitoid shows plasticity in exploiting volatile cues from various infested fruits, it is then capable of switching its preference patterns toward non-natal host fruits (or laboratory artificial substrate), which becomes also important for the development of rearing strategies for pest management programs (Biondi et al. 2017).

Using a chemical ecology approach, we here explored two ecological questions in our tritrophic study system. First, we investigated the effects of SWD infestation on the VOCs of ripening blueberries. Second, we addressed the behavioral response of female parasitoid wasps of a local population of $T$. anastrephae to fruits infested with this novel insect host.

\section{Methods And Materials}

\section{Fruits}

Organic grown blueberry fruits were used to analyze the effect of SWD attack on VOC profiles. The fruits were harvested weekly from multiple plants between December 2019 and January 2020 in a local organic farm (La Micaela, Canelones, Uruguay, http://lamicaelaorganico.com/). They were harvested before their fully ripen stage, still on the red stage as described by Gilbert et al. (2013) and Farneti et al. (2017) (see supplementary data Fig. S1). The fruits were harvested in the morning ( $9-12 \mathrm{am})$ and setup for VOC sampling in the laboratory the same afternoon.

Insects rearings 
Drosophila suzukii adults came from a laboratory colony established from flies collected locally in April 2019. The rearing was maintained on common cornmeal diet (504 ml distilled water, $66 \mathrm{~g}$ sucrose, $6 \mathrm{~g}$ bread yeast, $2.3 \mathrm{~g}$ agar, $52 \mathrm{~g}$ corn-flour, $1.3 \mathrm{ml}$ propionic acid, $0.8 \mathrm{~g}$ nipagin), in vial tubes (12 cm high, 2.5 $\mathrm{cm}$ diam.) placed in an incubator under controlled conditions $\left(21.5 \pm 1{ }^{\circ} \mathrm{C}, 65 \pm 5 \%\right.$ relative humidity, 12:12 $\mathrm{h}$ photoperiod). The parasitoids, $T$. anastrephae, also came from a laboratory rearing established at the same time (April 2019) from field-collected insects. These field collections represent the first report of the presence of this parasitoid in Uruguay. To work with wasps naive with respect to SWD, the parasitoid rearing was continuously maintained on $D$. melanogaster, under the same conditions as described above for SWD.

\section{Collection of Fruit Volatiles}

Blueberries free from external damages or irregularities ( $25 \mathrm{~g} \sim 15$ units) were placed in glass collecting chambers (20 cm length, $8 \mathrm{~cm}$ diam.) for lineal dynamic headspace volatile collection. VOCs were collected in passing air pushed from an air compressor at a flow rate of $0.5 \mathrm{I} \mathrm{min-1.} \mathrm{The} \mathrm{air} \mathrm{was}$ previously dehumidified with silica gel and filtered through activated carbon. VOCs were adsorbed in glass tubes filled with $50 \mathrm{mg}$ of HaySep Q (Alltech, USA) over a period of $24 \mathrm{~h}$. VOC collections were made at a temperature of $25 \pm 2{ }^{\circ} \mathrm{C}, 70 \pm 5 \%$ relative humidity and a photoperiod of $12: 12 \mathrm{~h}(\mathrm{~L}: \mathrm{D})$. Adsorbed volatiles were eluted with $1 \mathrm{ml}$ hexane, concentrated to $150 \mu \mathrm{l}$ under a gentle flow of $\mathrm{N} 2$, and stored in $250 \mu \mathrm{l}$ vial inserts at $-20^{\circ} \mathrm{C}$ until GC-MS analysis. An internal standard solution (100 $\mu$ l) was added prior to concentrating the sample (tridecane in hexane, $1 \mu \mathrm{g} / \mathrm{ml}$ ).

\section{Blueberry attacked by SWD females}

After the initial 24-h VOC collection the blueberry samples were assigned to the different treatments. VOC analyses resulting from these initial collections are shown as supplementary material (Fig. S2). To obtain SWD-attacked blueberries, the fruits were enclosed for $24 \mathrm{~h}$ with ten SWD couples ranging 2-7 days old. After $24 \mathrm{~h}$ the flies were anesthetized with $\mathrm{CO} 2$ and removed, and oviposition was confirmed under a stereo microscope by the presence of egg breathing tubes ( $9 \pm 6$ egg in each blueberry). The fruits were stored in clean glass containers covered with a fine mesh, under the same conditions as described for the insect rearing. Ten days later, a period that correlates with SWD egg to pupae development (Tochen et al. 2014), fruit VOCs were collected under the same conditions as described above (SWD-attacked samples). Two controls were performed to differentiate the effect of SWD attack on fruit VOCs from the effect of physical damage and the natural ripening of the fruit. Physical damage (Physically damage samples) was mimicked by gently punching all fruits in the $25 \mathrm{~g}$ cluster with a $5 \mu \mathrm{m}$ diam. microcapillary tube (three holes per fruit). The fruits were punctured ten days before VOC collection to match the maturation

time of SWD-attacked fruit. Natural ripening was allowed under the same conditions with no treatment as an additional control treatment (undamaged control samples). 


\section{Chemical Analyses}

Blueberry VOCs were analyzed by gas chromatography coupled with mass spectrometry (GC-MS) using a Shimadzu QP 2010 PLUS (Shimadzu Corp., Tokyo, Japan) equipped with a Rtx®-5MS column (30 m, $0.25 \mathrm{~mm}$ i.d, $0.25 \mathrm{~mm}$ film thickness; Alltech, USA). Samples $(1 \mu \mathrm{l})$ were injected in the splitless mode with He as carrier gas at a flow rate of $1 \mathrm{ml} / \mathrm{min}(49.7 \mathrm{KPa})$. The oven temperature was programmed from $40{ }^{\circ} \mathrm{C}$ for $4 \mathrm{~min}$, then increased to $150^{\circ} \mathrm{C}$ at $5^{\circ} \mathrm{C} / \mathrm{min}$ and to $250^{\circ} \mathrm{C}$ at $10^{\circ} \mathrm{C} / \mathrm{min}$ (held for $10 \mathrm{~min}$ ). Injector and MS transfer line temperatures were both set at $250^{\circ} \mathrm{C}$.

Volatile compounds were identified and quantified using the GCMS Solution software (Shimadzu GCMS Solution V 4.45SP1). The chromatograms were analyzed first by comparison with a blank run for background volatiles, then by comparison among the fruit VOC samples under the three treatments (SWD-attacked, physical damage, undamaged control). VOCs were identified from their mass spectra and retention indices, using the NIST08 and Adams' MS databases (Adams 2007). Individual compound net amounts were calculated relative to the internal standard by peak area comparison and are hence expressed as $\mu \mathrm{g}_{\text {int.std }}$ / $25 \mathrm{~g} / 24 \mathrm{~h}$.

\section{Olfactometer Bioassays}

The behavioral response of $T$. anastrephae females to volatiles from blueberries was evaluated using a glass Y-tube olfactometer consisting of two arms ( $6 \mathrm{~cm}$ long by $0.6 \mathrm{~cm}$ internal diameter) connected to chambers ( $9 \mathrm{~cm}$ long by $5 \mathrm{~cm}$ internal diameter) for the volatile stimuli. The chambers were located so that no visual contact was possible from the Y-tube. Humidified charcoal-filtered air was pushed through the stimuli and olfactometer with a pump at a rate of $0.5 \mathrm{l} / \mathrm{min}$. The olfactometer was laid horizontally on a glass surface homogeneously trans-illuminated with white cold LED lights (3600 lumens). To further eliminate visual cues, the olfactometer was fully enclosed in a box made from white corrugated plastic with a hole on top to allow video recording. All bioassays were conducted at $22 \pm 2{ }^{\circ} \mathrm{C}, 70 \pm 5 \% \mathrm{RH}$ and performed between 09:00 and 18:00 h. After each trial, the olfactometer arms were rotated to avoid position bias. At the end of the day the olfactometer was washed with distilled water and neutral soap, rinsed with ethanol and acetone and oven-dried at $100{ }^{\circ} \mathrm{C}$ for $24 \mathrm{~h}$.

T. anastrephae females were used 2-5 days after hatching from D. melanogaster pupae. Upon hatching, females and males were placed in glass containers with access to honey-water (50:50) absorbed in cotton balls, until their use in the experiments. To conduct the bioassay, each female wasp was individually placed at the base of the common section of the Y-tube, and a 5-min period was video recorded. From this video, we measured the time spent in each arm, the first arm selected, and the position at the end of the recording period. 
Three olfactometer experiments were performed: One bioassay tested undamaged blueberries vs clean air, the second tested SWD-attacked blueberries vs clean air, and the third compared SWD-attacked blueberries vs undamaged blueberries. For the two later experiments, $25 \mathrm{~g}$ of blueberries were exposed to oviposition by SWD under the same conditions as described for the VOC collections, and similarly kept for 10 days prior to the bioassays. Undamaged blueberries were stored under identical conditions. Blueberries for olfactometer bioassays were of commercial origin (Frusan, Frutera San Fernando, Chile); they were used fresh upon purchase and gently washed with distilled water.

\section{Statistical Analyses}

All statistical analyses were performed using R (Version 3.6-2) (R Core Development Team 2019).

To visualize VOC profiles in the different treatments a nonmetric multidimensional scaling (NMDS) was performed on the amount of VOCs matrix by using a Bray-Curtis distance matrix (Vegan package V2.4-6 in R). The data include many zero values, so it was fourth-root transformed (Hervé et al. 2018). To test significant differences in the chemical composition of VOCs produced among the treatments (SWDattacked, physical damage and control) a permutational multivariate analysis of variance (perMANOVA) was also carried out on the distance matrix based on 9999 permutations. The analysis was performed using the adonis function in R (Vegan package V2.4-6).

The behavioral response of parasitoid wasps was analyzed by a paired t-test for the time spent in each arm, and an exact binomial test both for the first arm chosen and the position at the end of the bioassay (five minutes). The exact binomial test performs an exact test of a simple null hypothesis about the probability that the number of wasps for first choice of either olfactometer arm had a 50:50 distribution. Also, to test the position at the end of the bioassay we performed the same test with a probability of 0.33 for the number of wasps present in either olfactometer arm or the common section. Females that did not respond were excluded from the analyses.

\section{Results}

\section{Effects of D. suzukii attack on blueberry VOC composition}

We measured VOCs from 15 samples of SWD-attacked blueberries, 10 samples of physically damaged fruit, and 15 samples of undamaged control. Twenty-four compounds were identified in the VOC extracts from blueberries (Table 1, Fig. 1, Table S1). Of these, nine compounds were exclusively present in the VOCs from blueberries attacked by $D$. suzukii (SWD-attacked) (Table 1). Taking into account the frequency in which it was found and the relative amount, isoamyl acetate was one of the main compounds that separates VOCs from SWD-attacked blueberries from VOCs of the fruit treatments (Table 1). Overall, volatiles produced in the highest amounts were short-chain aliphatic esters (Table 2). 
Multivariate analyses also showed that the VOC profiles of SWD-attacked blueberries differ from the VOC profiles of both control treatments (perMANOVA: $F_{2,37}=1.91 p=0.04$, permutation=9999). The NMDS ordination of the VOC composition partially separated SWD-attacked treatment from physically damaged and control VOCs (Fig. 2). Further, the multivariate pairwise comparation showed significant differences between the VOCs from SWD-attacked blueberries and those from physically attacked controls (perMANOVA: $F_{1,23}=2.63, p=0.03$, permutation=9999), as well from SWD-attacked blueberries and undamaged control fruit VOCs (perMANOVA: $F_{1,28}=2.26, p=0.04$, permutation=9999). Finally, the NMDS ordination did not graphically-show differences between VOCs from physically damaged and undamaged blueberries, nor did the multivariate analyses (perMANOVA: $F_{1,23}=0.34, p=0.83$, permutation=9999).

\section{Olfactory response of T. anastrephae to Blueberry volatiles}

Females of $T$. anastrephae reared on $D$. melanogaster responded preferentially to volatiles from blueberries attacked by $D$. suzukii, in comparison to undamaged blueberries or clean air. In both bioassays involving SWD-attacked fruits ( $v s$ air-control and $v s$ undamaged blueberries), about $60 \%$ of females made a choice during the 5-min test period (72 out of 109 and 53 out 92, respectively). Contrarily, in the bioassay contrasting undamaged control blueberries vs. clean air only around $30 \%$ of the females made a choice (28 out of 103). In general, the behavioral responses of $T$. anastrephae females showed a tendency to prefer the blueberry-treated arm. Parasitoid wasps were significantly attracted to SWDattacked blueberries when compared to clean air in all three variables measured (first choice binomial test $p=0.01$; final position binomial test $p=0.0003$ and time in each arm $t_{52}=2.52, p=0.01$ ) (Figs. 3-4). Also, $T$. anastrephae females were significantly attracted to the volatiles from SWD-infested blueberries when contrasted to undamaged blueberries when considering their final position (binomial test $p=$ 0.002 ), but not in the first arm choice (binomial test $p=0.1$ ) or the time spent in each olfactometer arm $\left(t_{71}=1.28, p=0.20\right)$. In the case of undamaged blueberries compared to clean air, there were no significant differences in any of the three variables measured (first choice binomial test $p=0.28$; final position binomial test $p=0.33$ and time in each arm $t_{27}=1.31, p=0.19$ ).

\section{Discussion}

The aim of our study was to characterize the effect of SWD attack on the VOCs of ripening blueberries, and to correlate these odor changes with the olfactory-mediated behavioral response of local populations of $T$. anastrephae female parasitoids. Our study is the first using a chemo-ecological approach to test the capacity of $T$. anastrephae to find $D$. suzukii-infested fruit.

Using fruit VOC chemical characterization followed by multivariate analyses of the odor blends of SWDattacked blueberries, we showed that the development of SWD immature stages inside the fruit generates 
a different odor profile. As we observed in our VOC analyses of maturing blueberries, fruits usually change their odor profiles during ripening. However, our results show that these changes are different in SWD-attacked blueberries, in comparison with physically damaged and undamaged control fruits. Just after field collection and before applying the treatments, VOCs were collected and analyzed and, as expected, they were homogenous among all samples (Fig. S2). Ten days after SWD infestation, a period that correlates with SWD larval development (Tochen et al. 2014), the odor differences among SWDattacked and control blueberries (physically damaged and undamaged) were significant and observable in the diversity, frequency and amounts of volatile compounds. SWD-related differences in the blueberry VOC profiles may be the result of various factors associated with SWD infestation, such as fruit tissue collapse due to larval feeding, larval metabolic wastes and their associated microorganisms, and opportunistic microorganisms associated with oviposition wounds (Hamby and Becher 2016, Rombaut et al. 2017).

Short-chain aliphatic esters, mostly represented by ethyl 3-methylbutanoate, was the most abundant chemical group in all three treatments. Along with the esters, the blueberries emitted sesqui- and monoterpenes as relatively minor components. Among the sesquiterpenes, delta-elemene was the most abundant and frequent, while limonene was the most abundant monoterpene in the control treatments. In SWD-attacked blueberries, however, the amount of limonene was similar to that of anhydrolinalool oxide, a probable fungal biotransformation metabolite. VOCs from SWD-attacked fruits also contained aromatic volatiles such as phenylethyl alcohol and ethyl benzoate, two common odorants from flowers and fruits that were not found in either of the control treatments. Besides these aromatic compounds, SWDattacked blueberries emitted seven additional compounds that were not found in the VOC extracts from both control treatments. Among these, the most abundant were 3-methylbutyl 3-methylbutanoate (isoamyl isovalerate) and isoamyl acetate. The latter was also the most consistently found among SWDattacked exclusive compounds.

Compounds such as hexanol, (Z)-linalool oxide and linalool are probably produced throughout ripening and preserved during the last maturation phases, reaching high amounts in the overripe stage (Horvat et al. 1996, Farneti et al. 2017). Other compounds seem to be emitted by unripe fruits and drastically reduced during ripening (i.e., caryophyllene), while compounds such as $\delta$-elemene are stable during all ripening phases (Farneti et al. 2017). In contrast, esters such as ethyl 3-methylbutanoate (ethyl isovalerate), ethyl acetate and methyl 2-methylbutanoate are exclusively produced in the last phase of ripening, increasing as the blueberries overripe (Farneti et al. 2017). In this scenario, our results suggest that SWD attack results in volatile emissions that resemble overripe fruit. 
Fruit volatiles are important in the chemical ecology of drosophilids and may play a role in niche differentiation among sympatric species. Even though cosmopolitan Drosophila species are host generalists, it is possible that different species separate along resource-based niche dimensions such as fruit maturation time (Nunney 1996). SWD may be attracted to leaf volatiles cues to mate-finding and also fresh unripe fruits odors to locate areas for oviposition (Cloonan et al. 2018). As fruit ripen, other drosophilids such as Drosophila simulans, D. melanogaster and Drosophila immigrans may further colonize this rotten fruit, following a preference order for increasing maturation stages (Atallah et al. 2014, Rombaut et al. 2017, Nunney, 1996). Fruit volatile esters may provide cues for ripening stages and may encode enough information to enable drosophilid flies to detect and discriminate their niches (Scheidler et al. 2015). For instance, isoamyl acetate, a "fruity" odour often present in ripening, ripe and early fermenting fruits, is known to attract many drosophilids (Stökl et al. 2010). This compound, along with isobutyl acetate and ethyl hexanoate, were present in headspace VOC samples of fruit-associated yeasts and caused antennal responses in D. melanogaster and D. suzukii (Scheidler et al. 2015). Moreover, isoamyl acetate was one of the EAD-active compounds from wild blueberries, attractive to $D$. suzukii both individually and as part of a blend (Urbaneja-Bernat et al. 2021). However, tested in formulated blends for trapping SWD, isoamyl acetate showed no attraction and even a decrease in SWD adult captures (Cha et al. 2012). In this case, it is possible that concentration modulates SWD response to volatiles such as isoamyl acetate, since high concentrations may signal an overripe fruit that is not a preferred oviposition site for SWD females (Revadi et al. 2015).

The dynamics of fruit volatile blends associated with the temporal separation of Drosophila species may provide host-finding cues to the next trophic level (Vet and Dicke 1992). Using behavioral bioassays, we demonstrated that resident populations of $T$. anastrephae, a pupal parasitoid reared in the laboratory on D. melanogaster, responded differentially to volatiles emitted by blueberries infested by SWD. We measured three variables to characterize female wasp responses to the volatiles of blueberries; the first choice, the time spent in each arm, and the final position after five minutes of bioassay. Females of $T$. anastrephae showed a preference in all three variables when SWD-attacked fruits were tested against clean air. When the bioassay compared SWD-attacked and undamaged blueberries, a preference was only found in the position of the wasp at the end of the tested period, a variable that may correlate with active seeking behaviour of the preferred source. These results are in line with those obtained from a related parasitoid, T. drosophilae. Using an olfactometer arena with four chambers and testing seven wild noncrop fruits as stimuli, Wolf et al. (2020) showed that females spent more time walking over chambers with SWD-infested fruits compared to clean air. When comparing SWD-infested fruits with non-infested fruits significant preferences were not so consistent, indicating that fruit odours alone may not be not sufficient for host location in these fruits (Wolf et al. 2020). The preference showed by $T$. anastrephae females towards volatiles of SWD-infested blueberries appear to be innate, since they have had no access to SWD or SWD-infested fruit prior to the tests. SWD infestation of blueberries resulted in an increase of typical volatile compounds that other Drosophila species use for locating food, mating and oviposition sites. It is then likely that $T$. anastrephae females use these same general odorant cues to 
locate their established drosophilid hosts. The foraging females may switch hosts if other alternatives are more abundant (Jaworski et al. 2013) or if they find earlier infested fruits that are more likely to contain pupae. In support of this, the generalist parasitoid T. drosophilae showed no differential preference for $D$. melanogaster or $D$. suzukii pupae (Wang et al. 2016). Similarly, a recent study by Biondi et al. (2020) showed that the larval parasitoids, Asobara japonica Belokobylskij (Hymenoptera: Braconidae), Leptopilina japonica Novković \& Kimura and Ganaspis brasiliensis (Ihering) (Hymenoptera: Figitidae), respond to fruit volatile cues associated with the presence of either $D$. suzukii or $D$. melanogaster (Biondi et al. 2020).

From an applied perspective, our results represent a relevant contribution to the development of a biological control program for SWD, since it deepens our understanding of how SWD impact blueberry VOCs, and how a parasitoid responds to the presence of its potential pest host (Biondi et al. 2017). Our study highlights the potential use of established populations of $T$. anastrephae since they are naturally able to cue on VOCs from SWD-attacked fruit and use SWD as a viable host (Vieria et al. 2020). Although most SWD pupae are found in the soil (Wolz et al. 2017), by using fruit VOCs as a long-range cue, parasitoids could target the right ecological niche to then refine their search. Understanding the behavior of this parasitoid in challenging environments is important to obtain higher efficiency in biological control programs against SWD (Krüger et al. 2019), stressing the need of more studies on the biology of $T$. anastrephae in the region. In line with this, the absence of management in adjacent crops or native fruits could be reservoir of SWD where resident populations of parasitoids could have a greater importance. The parasitoid is already established and well adapted to local environmental conditions, which further underlines its potential as a biological control agent also in unmanaged adjacent crops or nearby native fruits, which could serve as reservoirs for SWD (Krüger et al. 2019). In a broader sense, studies on these local parasitoid populations at the regional level may offer opportunities to manage SWD in Latin America without new introduction of exotic species. Hence, a nonnative organism such as SWD in Latin America may become controlled by a regulating mechanism in the introduced range, which limits its density and expansion (Schulz et al. 2019).

\section{Declarations}

\section{Fundings}

This project was funded by grants from Agencia Nacional de Investigación e Innovación (ANII): FMV-12019-1-156089 and PD_NAC_2018_1_150632 (fellowship to GD).

\section{Conflict of interest}

All authors declare that they have no conflict of interest.

\section{Availability of data and material}


Data is available from the corresponding authors on request.

\section{Code availability}

Code is available from the corresponding authors on request.

\section{Author contributions}

$A G$ and $G D$ conceived and designed research; $G D, A G$ and FT collected the data; $A G$ identified the chemical compounds; FT led the parasitoid experiments; GD and AG led the analysis and writing of the manuscript. All authors contributed to the drafts and approved the manuscript.

\section{Acknowledgements}

This project was funded by grants from Agencia Nacional de Investigación e Innovación: FMV-1-2019-1156089 and PD_NAC_2018_1_150632 (fellowship to GD). We thank Dr. Beatriz Goñi for kind help and advice in field collections, Daniela Mato for assistance in VOC collections, and Finca La Micaela for access to their organic farm.

\section{References}

Adams RP (2007) Identification of essential oil components by gas chromatography/ mass spectrometry, 4th Edition. Allured Publ., Carol Stream, IL

Anderson PK, Cunningham AA, Patel NG et al (2004) Emerging infectious diseases of plants: pathogen pollution, climate change and agrotechnology drivers. Trends Ecol Evol 19(10):535-544. https://doi.org/10.1016/j.tree.2004.07.021

Atallah J, Teixeira L, Salazar R, Zaragoza G, Kopp A (2014) The making of a pest: the evolution of a fruitpenetrating ovipositor in Drosophila suzukii and related species. Proc Biol Sci. 281(1781):20132840. doi: 10.1098/rspb.2013.2840.

Biondi A, Wang X, Daane KM (2020) Host preference of three Asian larval parasitoids to closely related Drosophila species: implications for biological control of Drosophila suzukii. J Pest Sci doi.org/10.1007/s10340-020-01272-0 
Biondi A, Wang X, Miller JC et al (2017) Innate olfactory responses of Asobara japonica toward fruits infested by the invasive spotted wing drosophila. J Insect Behav 30:495-506. https ://doi.org/10.1007/s1090 5-017-9636-y

Bolda MP, Goodhue RE, Zalom FG (2010) Spotted wing drosophila: potential economic impact of newly established pest. Giannini Foundation of Agricultural Economics, University of California. Agric Resour Econ Update 13(3):5-8

Cha D, Adams T, Rogg H, Landolt PJ (2012) Identification and Field Evaluation of Fermentation Volatiles from Wine and Vinegar that Mediate Attraction of Spotted Wing Drosophila, Drosophila suzukii. J Chem Ecol (2012) 38:1419-1431

Chabaane Y, Laplanche D, Turlings TC, Desurmont GA (2015) Impact of exotic insect herbivores on native tritrophic interactions: a case study of the African cotton leafworm, Spodoptera littoralis and insects associated with the field mustard Brassica rapa. Journal of Ecology, 103(1), 109-117.

Cini A, loriatti C, Anfora G (2012) A review of the invasion of Drosophila suzukii in Europe and a draft research agenda for integrated pest management. $B$ Insectol 65(1):149-160

Cloonan KR, Abraham J, Angeli S et al (2018) Advances in the Chemical Ecology of the Spotted Wing Drosophila (Drosophila suzukii) and its Applications. J Chem Ecol 44, 922-939. https://doi.org/10.1007/s10886-018-1000-y

Cusumano A, Harvey JA, Bourne ME, Poelman EH, de Boer JG (2020) Exploiting chemical ecology to manage hyperparasitoids in biological control of arthropod pests. Pest management science, 76(2), 432443.

de la Vega GJ, Corley JC (2019) Drosophila suzukii (Diptera: Drosophilidae) distribution modelling improves our understanding of pest range limits. J. Pest Manage, Int. https://doi.org/10.1080/09670874.2018.1547460 
de la Vega GJ, Corley JC Soliani C (2019) Genetic assessment of the invasion history of Drosophila suzukii in Argentina. J Pest Sci. 93, 63-75. https://doi.org/10.1007/s10340-019-01149-x

Desurmont GA, von Arx M, Turlings TCJ, Schiestl FP (2020) Floral Odors Can Interfere With the Foraging Behavior of Parasitoids Searching for Hosts. Front. Ecol. Evol. 8:148. doi: 10.3389/fevo.2020.00148.

Farneti B, Khomenko I, Grisenti M et al. (2017) Exploring blueberry aroma complexity by chromatographic and direct-injection spectrometric techniques. Frontiers in plant science, 8, 617.

Gilbert JL, Schwieterman ML, Colquhoun TA, Clark DG, Olmstead JW (2013) Potential for increasing southern highbush blueberry flavor acceptance by breeding for major volatile components. HortScience, 48(7), 835-843.

Hamby KA, Becher PG (2016) Current knowledge of interactions between Drosophila suzukii (Diptera: Drosophilidae) and microbes, and their potential utility for pest management. J Pest Sci. 89(3), 621-630.

Harvey JA, Fortuna TM (2012) Chemical and structural effects of invasive plants on herbivoreparasitoid/predator interactions in native communities. Entomologia Experimentalis et Applicata, 144, 1426.

Hervé MR, Nicolè F, Lê Cao K (2018) Multivariate Analysis of Multiple Datasets: a Practical Guide for Chemical Ecology. J Chem Ecol 44, 215-234. https://doi.org/10.1007/s10886-018-0932-6

Horvat RJ, Schlotzhauer WS, Chortyk OT et al (1996) Comparison of volatile compounds from rabbiteye blueberry (Vaccinium ashei) and deerberry (V. stamineum) during maturation. J. Essent. Oil Res. 8, 645-648. 
Ibouh K, Oreste M, Bubici G, et al (2019) Biological control of Drosophila suzukii: Efficacy of parasitoids, entomopathogenic fungi, nematodes and deterrents of oviposition in laboratory assays. Crop Protection, 125, 104897.

Jaworski CC, Bompard A, Genies L, Amiens-Desneux E, Desneux N (2013) Preference and Prey Switching in a Generalist Predator Attacking Local and Invasive Alien Pests. PLoS ONE 8(12): e82231.

Karageorgi M, Brńcker LB, Lebreton S et al (2017) Evolution of multiple sensory systems drives novel egglaying behavior in the fruit pest Drosophila suzukii. Current Biology, 27(6), 847-853.

Keesey IW, Knaden M, Hansson BS (2015) Olfactory specialization in Drosophila suzukii supports an ecological shift in host preference from rotten to fresh fruit. Journal of Chemical Ecology, 41(2), 121-128.

Krüger AP, Scheunemann T, Vieira JGA, et al (2019) Effects of Extrinsic, Intraspecific Competition and Host Deprivation on the Biology of Trichopria anastrephae (Hymenoptera: Diapriidae) Reared on Drosophila suzukii (Diptera: Drosophilidae). Neotrop Entomol 48(6):957-965. doi: 10.1007/s13744-01900705-5.

Kruitwagen A, Beukeboom LW, Wertheim B (2018) Optimization of native biocontrol agents, with parasitoids of invasive pest Drosophila suzukii as an example. Evolutionary Applications. 11:1473-1497.

Lantschner MV, de la Vega GJ, Corley JC (2019) Modelling the establishment, spread and distribution shifts of pests. Int J Pest Manage 1:1. https://doi.org/10.1080/09670874.2019.1575490

Lee, JC, Wang X, Daane KM, Hoelmer KA, Isaacs R, Sial AA, Walton VW (2019). Biological Control of Spotted-Wing Drosophila (Diptera: Drosophilidae)-Current and Pending Tactics. J. Integr. Pest Manag. 10:1, 22. 
Liu CM, Matsuyama S, Kainoh Y (2019) Synergistic Effects of Volatiles from Host-Infested Plants on Host-Searching Behavior in the Parasitoid Wasp Lytopylus rufipes (Hymenoptera: Braconidae). Journal of chemical ecology, 45(8), 684-692.

Mair MM, Ruther J (2019) Chemical ecology of the parasitoid wasp genus Nasonia (Hymenoptera, Pteromalidae). Frontiers in Ecology and Evolution, 7, 184.

Mumm R, Hilker M (2005) The significance of background odour for an egg parasitoid to detect plants with host eggs. Chem Senses 30:337-343.

Nunney L (1996) The Colonization of Oranges by the Cosmopolitan Drosophila. Oecologia. 108 (3), $552-$ 561.

R Core Development Team (2019) R: a language and environment for statistical computing. R Foundation for Statistical Computing, Vienna.

Revadi S, Vitagliano S, Rossi Stacconi MV, Ramasamy S, Mansourian S, et al (2015) Olfactory responses of Drosophila suzukii females to host plant volatiles. Physiological Entomology, 40(1), 54-64.

Rombaut A, Guilhot R, Xuéreb A, Benoit L, Chapuis MP, Gibert P, Fellous S (2017) Invasive Drosophila suzukii facilitates Drosophila melanogaster infestation and sour rot outbreaks in the vineyards. $\mathrm{R}$. Soc.open sci. 4: 170117. http://dx.doi.org/10.1098/rsos.170117

Scheidler NH, Liu C, Hamby KA, Zalom FG, Syed Z (2015) Volatile codes: correlation of olfactory signals and reception in Drosophila-yeast chemical communication. Scientific reports, 5, 14059.

Schröder R, Hilker M (2008) The relevance of background odor in resource location by insects: a behavioral approach. AIBS Bull. 58, 308-316. 
Schulz AN, Lucardi RD, Marsico TD (2019) Successful Invasions and Failed Biocontrol: The Role of Antagonistic Species Interactions, BioScience, 69(9), 711-724 https://doi.org/10.1093/biosci/biz075.

Stökl J, Strutz A, Dafni A et al (2010) A deceptive pollination system targeting drosophilids through olfactory mimicry of yeast. Current Biology, 20(20), 1846-1852.

Tochen S, Dalton DT, Nik W et al (2014) Temperature-Related Development and Population Parameters for Drosophila suzukii (Diptera: Drosophilidae) on Cherry and Blueberry. Environmental Entomology. 43:2 501-510.

Vet LEM, Dicke M 1(992) Ecology of infochemical use by natural enemies in a tritrophic context. Annual Review of Entomol. 37:141-172.

Vieira JGA, Krüger AP, Scheunemann T, et al (2020) Effect of temperature on the development time and life-time fecundity of Trichopria anastrephae parasitizing Drosophila suzukii. Appl Entomol. 00:1-9. DOI: 10.1111/jen.12799.

Vieira JGA, Krüger AP, Scheuneumann T, et al (2019) Some aspects of the biology of Trichopria anastrephae (Hymenoptera: Diapriidae), a resident parasitoid attacking Drosophila suzukii (Diptera: Drosophilidae) in Brazil. Journal of Economic Entomology, 113, 81-87.

Urbaneja-Bernat P, Cloonan K, Zhang A. et al. (2021). Fruit volatiles mediate differential attraction of Drosophila suzukii to wild and cultivated blueberries. J Pest Sci (2021). https://doi.org/10.1007/s10340021-01332-z

Walsh DB, Bolda MP, Goodhue RE, Dreves AJ, Lee J, Bruck DJ (2011) Drosophila suzukii (Diptera: Drosophilidae): invasive pest of ripening soft fruit expanding its geographic range and damage potential. J Integr Pest Manag. 2:1-8 
Wang X, Kacar G, Biondi A, Daane KM (2016) Life-history and host preference of Trichopria drosophilae, a pupal parasitoid of spotted wing drosophila. BioControl 61:387-397.

Woltz J, Megan J, Lee C (2019). Pupation behavior and larval and pupal biocontrol of Drosophila suzukii in the field. Biological control 110 (2017): 62-69.

Wolf S, Boycheva-Woltering S, Romeis J, Collatz J (2020) Trichopria drosophilae parasitizes Drosophila suzukii in seven common non-crop fruits. J Pest Sci 93, 627-638.

Wollmann J, Schlesener DCH, Ferreira MS, Garcia FRM (2016) Parasitoids of Drosophilidae with potential for parasitism on Drosophila suzukii in Brazil. Drosophila Information Service, 99, 38-42.

\section{Tables}

Table 1. Volatile Organic Compounds (VOCs) found in the volatile extracts from blueberries subjected to the different treatments: attacked by Drosophila suzukii (SWD-attacked); physical damage control and natural fruit ripening (undamaged control). VOC components are classified in groups as short-chainaliphatic esters (SCA-est), sesquiterpenes, monoterpene, aromatics and others. Amount values are shown as $\mu \mathrm{g}_{\text {int.std. }} / 25 \mathrm{~g} / 24 \mathrm{~h}$ (mean \pm standard deviation), ( $\mathrm{n}$ ) indicates the number of samples in which a compound was found; (\#) indicates compound numbers as in Figure 1 and Table S1. See methods for details about volatile quantification and Table S1 for identification details. 


\begin{tabular}{|c|c|c|c|c|c|c|c|c|}
\hline Group & $\#$ & Compound & $\begin{array}{l}\text { SWD- } \\
\text { attacked }\end{array}$ & (n) & $\begin{array}{l}\text { Physical } \\
\text { damage }\end{array}$ & (n) & $\begin{array}{l}\text { Undamaged } \\
\text { control }\end{array}$ & (n) \\
\hline \multirow[t]{11}{*}{ SCA-est } & 1 & ethyl isobutyrate & $\begin{array}{l}0.025 \pm \\
0.03\end{array}$ & 3 & 0.021 & 1 & 0.005 & 1 \\
\hline & 2 & $\begin{array}{l}\text { methyl 3- } \\
\text { methylbutanoate }\end{array}$ & $\begin{array}{l}0.503 \pm \\
1.15\end{array}$ & 8 & $\begin{array}{l}0.341 \pm \\
0.09\end{array}$ & 2 & $\begin{array}{l}0.125 \pm \\
0.10\end{array}$ & 4 \\
\hline & 3 & ethyl butanoate & 0.257 & 1 & 0.149 & 1 & & \\
\hline & 4 & $\begin{array}{l}\text { methyl 3-methyl-2- } \\
\text { butenoate }\end{array}$ & $\begin{array}{l}0.073 \pm \\
0.03\end{array}$ & 4 & & & & \\
\hline & 5 & $\begin{array}{l}\text { ethyl } 2 \text { - } \\
\text { methylbutanoate }\end{array}$ & $\begin{array}{l}0.167 \pm \\
0.15\end{array}$ & 15 & $\begin{array}{l}0.097 \pm \\
0.07\end{array}$ & 10 & $\begin{array}{l}0.143 \pm \\
0.10\end{array}$ & 15 \\
\hline & 6 & $\begin{array}{l}\text { ethyl 3- } \\
\text { methylbutanoate }\end{array}$ & $\begin{array}{l}2.559 \pm \\
3.45\end{array}$ & 15 & $\begin{array}{l}0.973 \pm \\
1.31\end{array}$ & 10 & $\begin{array}{l}1.549 \pm \\
1.54\end{array}$ & 15 \\
\hline & 8 & isoamyl acetate & $\begin{array}{l}0.144 \pm \\
0.09\end{array}$ & 7 & & & & \\
\hline & 9 & $\begin{array}{l}\text { isopropyl 3- } \\
\text { methylbutanoate }\end{array}$ & $\begin{array}{l}0.020 \pm \\
0.01\end{array}$ & 5 & $\begin{array}{l}0.034 \pm \\
0.03\end{array}$ & 2 & $\begin{array}{l}0.037 \pm \\
0.02\end{array}$ & 2 \\
\hline & 10 & $\begin{array}{l}\text { ethyl 3-methyl-2- } \\
\text { butenoate }\end{array}$ & $\begin{array}{l}0.112 \pm \\
0.08\end{array}$ & 2 & 0.045 & 1 & $\begin{array}{l}0.036 \pm \\
0.00\end{array}$ & 2 \\
\hline & 15 & $\begin{array}{l}\text { 3-methylbutyl 2- } \\
\text { methylbutanoate }\end{array}$ & 0.03 & 1 & & & & \\
\hline & 16 & $\begin{array}{l}\text { 3-methylbutyl 3- } \\
\text { methylbutanoate }\end{array}$ & $\begin{array}{l}0.293 \pm \\
0.10\end{array}$ & 2 & & & & \\
\hline \multirow[t]{5}{*}{ sesquiterp. } & 19 & delta-elemene & $\begin{array}{l}0.182 \pm \\
0.12\end{array}$ & 12 & $\begin{array}{l}0.269 \pm \\
0.15\end{array}$ & 9 & $\begin{array}{l}0.246 \pm \\
0.21\end{array}$ & 13 \\
\hline & 20 & caryophyllene & $\begin{array}{l}0.003 \pm \\
0.003\end{array}$ & 4 & $\begin{array}{l}0.008 \pm \\
0.01\end{array}$ & 2 & $\begin{array}{l}0.014 \pm \\
0.02\end{array}$ & 4 \\
\hline & 21 & cis-Thujopsene & $\begin{array}{l}0.015 \pm \\
0.01\end{array}$ & 6 & $\begin{array}{l}0.002 \pm \\
0.001\end{array}$ & 2 & 0.006 & 1 \\
\hline & 23 & $\begin{array}{l}\text { 4,5-di-epi- } \\
\text { aristolochene }\end{array}$ & $\begin{array}{l}0.037 \pm \\
0.02\end{array}$ & 2 & & & & \\
\hline & 24 & trans-beta-Guaiene & $\begin{array}{l}0.007 \pm \\
0.005\end{array}$ & 4 & $\begin{array}{l}0.009 \pm \\
0.01\end{array}$ & 4 & $\begin{array}{l}0.014 \pm \\
0.01\end{array}$ & 6 \\
\hline \multirow[t]{3}{*}{ monoterp. } & 11 & $\begin{array}{l}\text { anhydrolinalool } \\
\text { oxide }\end{array}$ & $\begin{array}{l}0.090 \pm \\
0.04\end{array}$ & 3 & 0.049 & 1 & $\begin{array}{l}0.043 \pm \\
0.01\end{array}$ & 3 \\
\hline & 12 & limonene & $\begin{array}{l}0.093 \pm \\
0.15\end{array}$ & 11 & $\begin{array}{l}0.087 \pm \\
0.08\end{array}$ & 4 & $\begin{array}{l}0.133 \pm \\
0.23\end{array}$ & 9 \\
\hline & 13 & cineole & $\begin{array}{l}0.032 \pm \\
0.01\end{array}$ & 3 & & & & \\
\hline
\end{tabular}




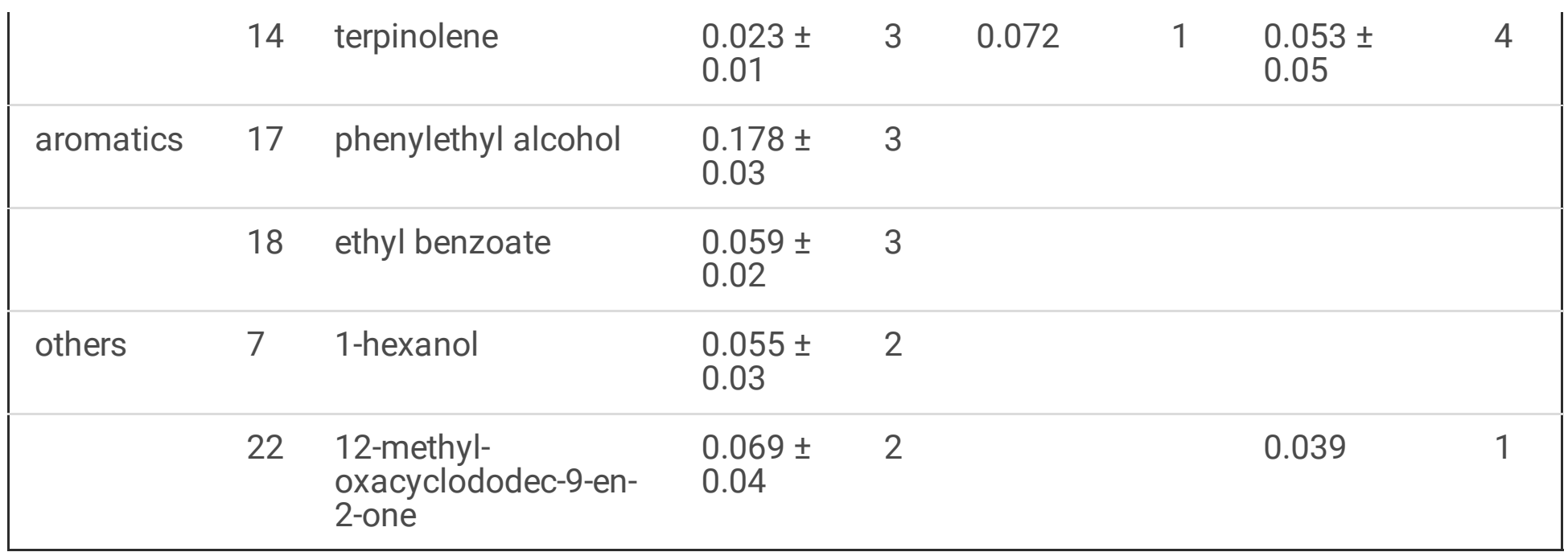

Table 2. Blueberry volatile organic compounds (VOCs) clustered by chemical group, according to fruit treatments: attacked by Drosophila suzukii (SWD-attacked); physical damage control and natural fruit ripening (undamaged control). SCA-Esters stands for short-chain aliphatic esters. See Table 1 and methods section for details. Amounts are expressed as $\mu \mathrm{g}_{\text {int.std. }} / 25 \mathrm{~g} / 24 \mathrm{~h}$ (mean \pm standard deviation). (n) Indicates the number of samples in which compounds of the chemical group were found.

\begin{tabular}{|lllllll|}
\hline Compound group & SWD-attacked & $(\mathbf{n})$ & Physical damage & $(\mathbf{n})$ & Undamaged control & $(\mathbf{n})$ \\
\hline SCA-Esters & $3.17 \pm 4.63$ & 15 & $1.17 \pm 1.52$ & 10 & $1.74 \pm 1.61$ & 15 \\
\hline sesquiterpenes & $0.17 \pm 0.13$ & 14 & $0.28 \pm 0.16$ & 9 & $0.24 \pm 0.22$ & 14 \\
monoterpenes & $0.12 \pm 0.15$ & 12 & $0.12 \pm 0.14$ & 4 & $0.17 \pm 0.29$ & 9 \\
\hline aromatics & $0.14 \pm 0.09$ & 5 & & & & \\
\hline others & $0.06 \pm 0.03$ & 4 & & & 0.04 & 1 \\
\hline
\end{tabular}

\section{Figures}




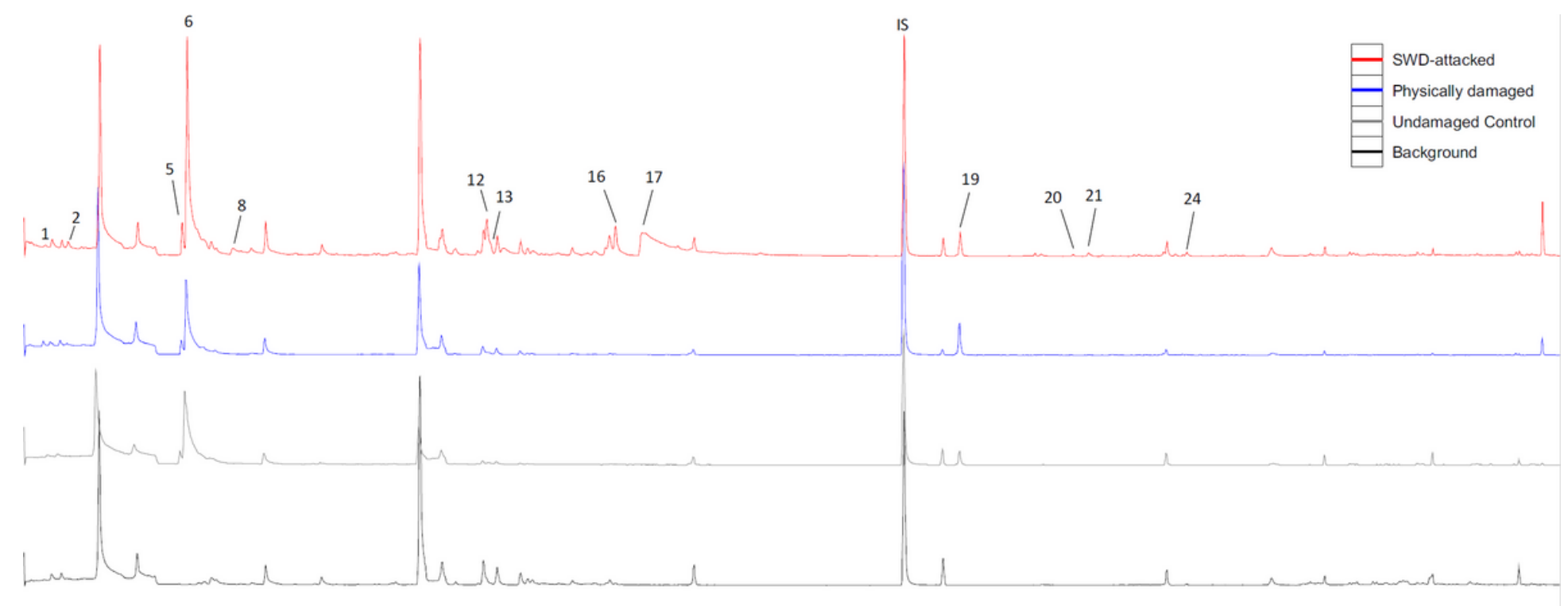

Figure 1

Typical chromatogram (TIC) traces of VOC extracts from blueberries attacked by SWD (red), physically damaged control (blue), undamaged control (grey) and ambient background volatiles (black). Numbers indicate compounds as described in Tables 1 and S1. Non-numbered peaks correspond to background volatiles. Missing numbers are blueberry VOCs not found in the samples chosen for the figure. IS stands for internal standard. 


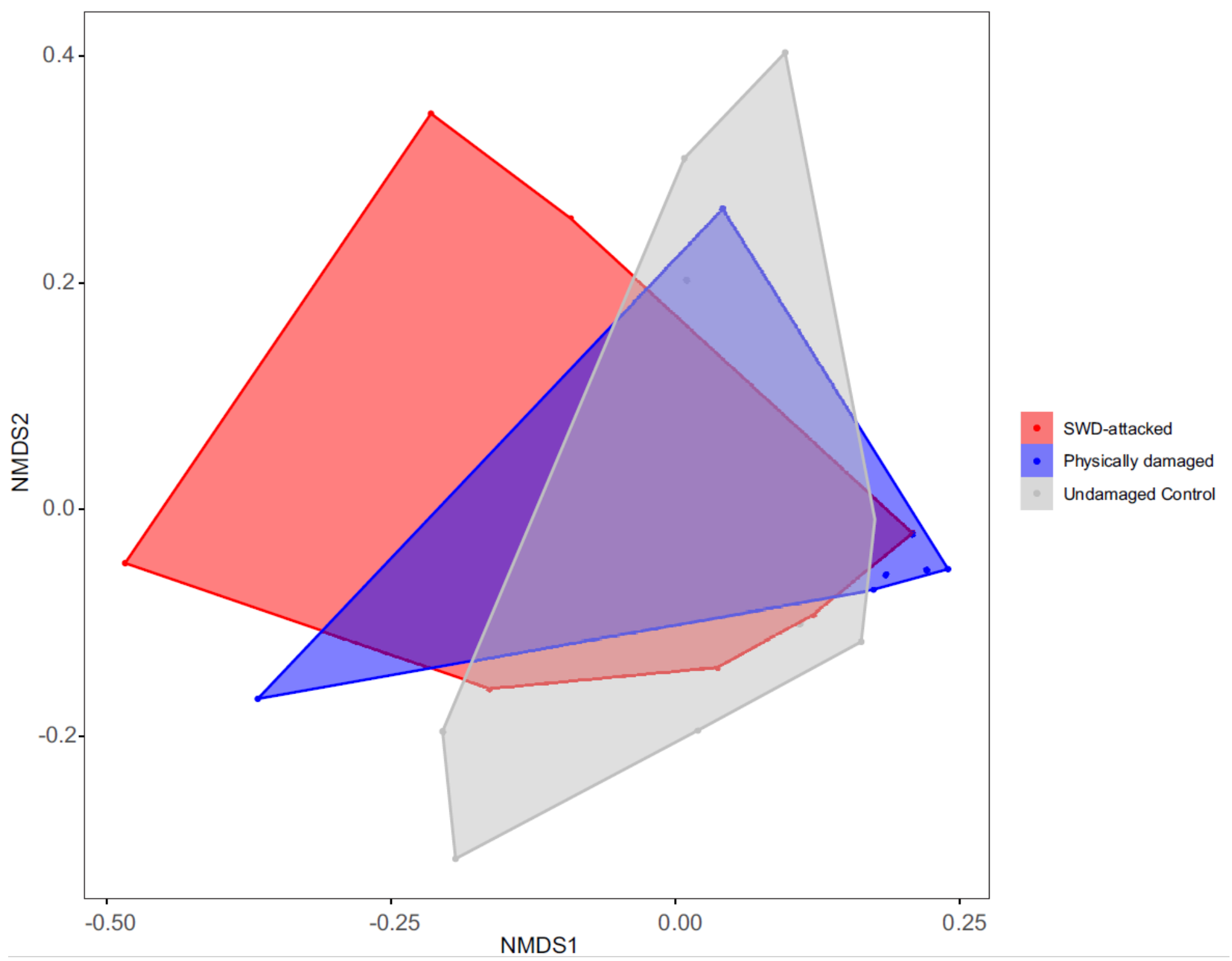

Figure 2

Non-metric multidimensional scaling (NMDS) ordination based on Bray-Curtis dissimilarities of the volatile organic compounds (VOCs) from blueberries attacked by Drosophila suzukii (SWD-attacked, $n=$ 15); VOCs from physically-damaged blueberries $(n=10)$ and from undamaged fruit (Control, $n=15$ ). Stress value $=0.1615$. 


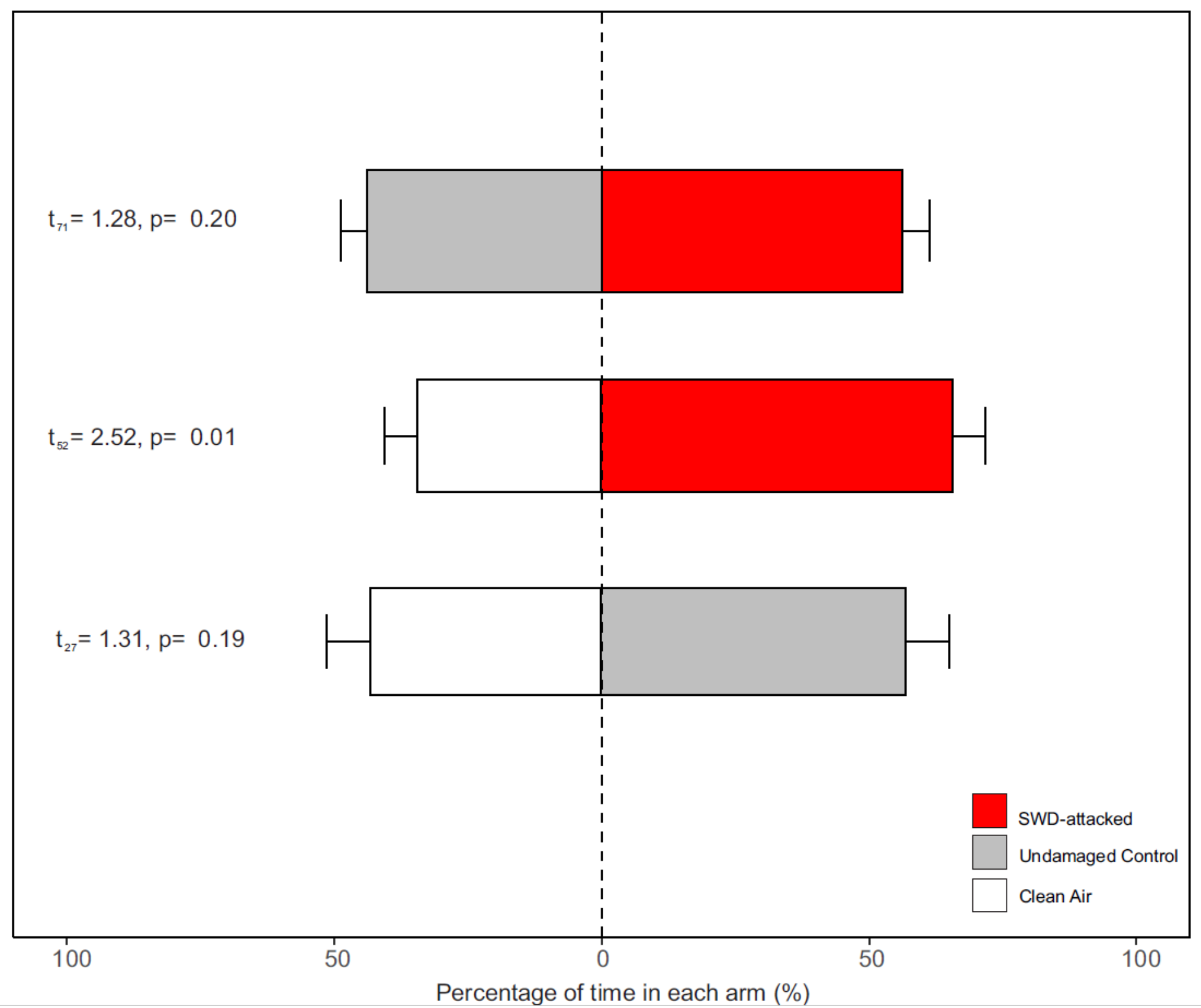

Figure 3

Percent time spent by Trichopria anastrephae females in each arm of an olfactometer tube in choice bioassays testing volatile cues produced by blueberries attacked by Drosophila suzukii (SWD-attacked), control blueberries (undamaged) or clean air. Paired t-test are reported and error bars show Standard Error. 


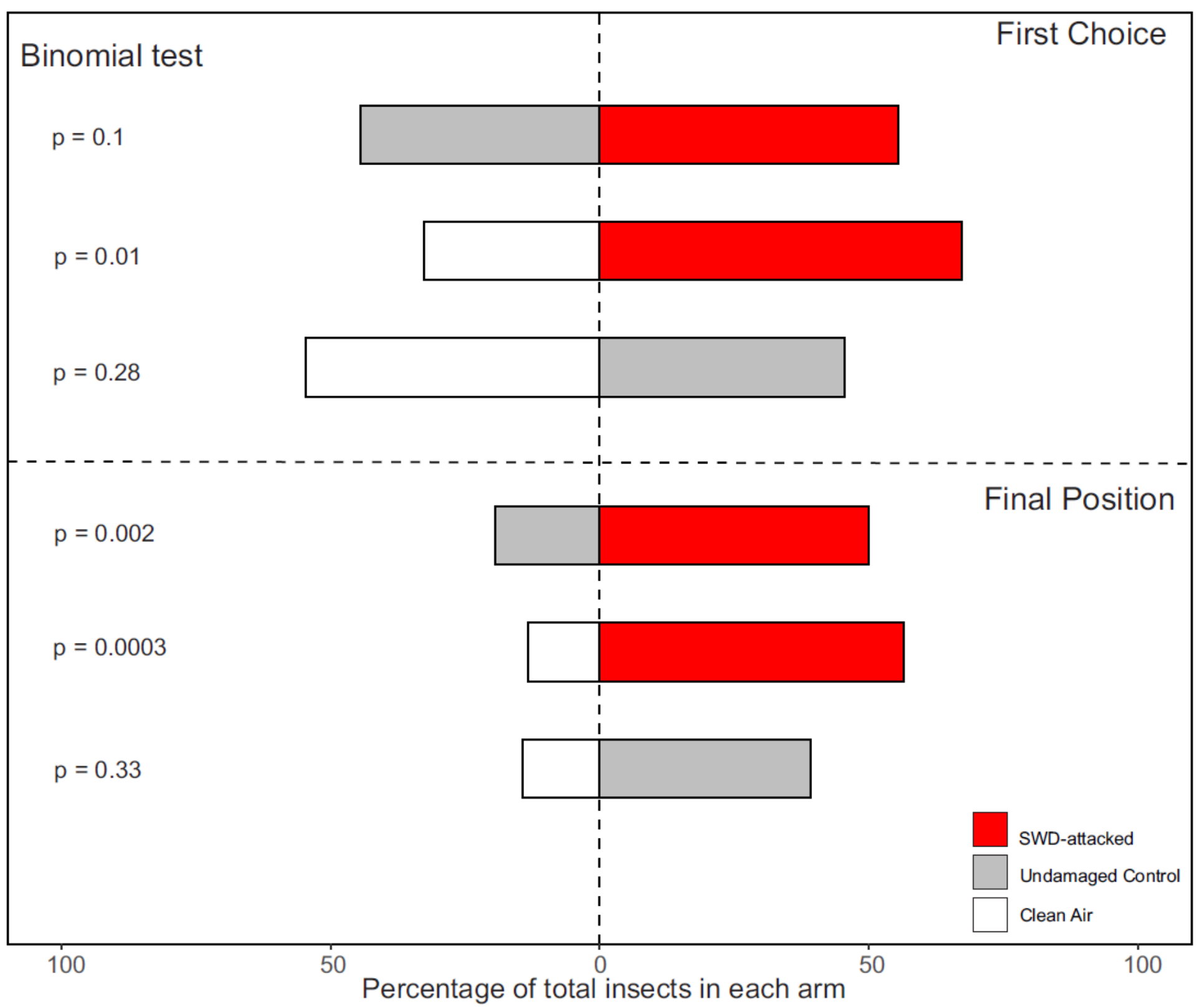

Figure 4

Percent Trichopria anastrephae females in each arm of an olfactometer tube in choice bioassays with volatile cues produced by blueberries attacked by Drosophila suzukii (SWD-attacked), undamaged control or clean air. The upper and lower panels show the first arm choice and the the final position at the end of a 5 -min test period, respectively. p-Values for the binomial test are reported.

\section{Supplementary Files}

This is a list of supplementary files associated with this preprint. Click to download.

- SupMatTableS1.xlsx

- SupMatdelaVega2021JCE.doc 